\title{
Relazione suolo, pianta, atmosfera: sicurezza e qualità delle produzioni agroalimentari e tutela dell'ambiente
}

L'agricoltura può essere rappresentata come punto di intersezione fra aspetti biofisici (piante, animali, microrganismi, terreno, acqua, atmosfera ecc.), tecnico economici (produzioni, tecnologie, mezzi tecnici, mercati, prezzi ecc.) e socio-politici (popolazioni, culture, istituzioni politiche ecc.). I problemi che un sistema siffatto pone alla ricerca sono di conseguenza di grande complessità soprattutto nel momento in cui si vogliano perseguire soluzioni rispettose della qualità dei prodotti della sicurezza dei consumatori e in armonia con l'ambiente. Queste problematiche presentano una vera e propria sfida per i ricercatori che operano nel settore agricolo e che si trovano di fronte ad un sistema che deve essere analizzato con il più elevato livello di specializzazione disciplinare possibile senza però perderne di vista l'unitarietà e la complessità. L'avanzamento delle conoscenze e l'individuazione delle migliori soluzioni tecniche risultano pertanto strettamenti legati ad un approccio multidisciplinare che preveda l'indagine a tutti i livelli di scale spazio-temporali coinvolgenti una problematica.

Sulla base di queste premesse l'Associazione Italiana Società Scientifiche Agrarie (AISSA), in collaborazione con la Facoltà di Agraria dell'Università di Foggia, organizza il suo V Convegno annuale che verterà sul tema: "Relazione suolo, pianta, atmosfera: sicurezza e qualità delle produzioni agroalimentari e tutela dell'ambiente".

Il Convegno, articolato in sessioni nelle qua- li verranno presentati i contributi che le diverse Società Scientifiche che costituiscono l'AISSA stanno fornendo per lo studio e la soluzione di specifiche problematiche, deve essere inteso come un tentativo di fornire una visione il più possibile integrata relativamente all'argomento, che coinvolge aspetti di rilevante interesse per il mondo produttivo e che riguardano l'uso ottimale delle risorse biofisiche, l'impatto dei cambiamenti climatici, il contributo del sistema agroindustriale ai problemi energetici e i rapporti fra alimenti e salute. Ogni sessione scientifica sarà completata dalla presenza di poster dando così la possibilità ai diversi gruppi di ricerca di far conoscere il loro lavoro e di discuterlo anche con ricercatori appartenenti ad ambienti culturali diversi, o che operino nell'ambito di una stessa tematica su scale spazio-temporali diverse.

Si auspica che un Convegno così impostato possa attirare l'attenzione non solo di ricercatori, studenti e tecnici di enti pubblici e privati ma anche di imprenditori della filiera agroalimentare interessati ad un aggiornamento multidisciplinare e con i quali è indispensabile consolidare un rapporto tale da valorizzare l'intenso lavoro scientifico che con impegno, in periodi non facili per la ricerca italiana, viene portato avanti dai ricercatori.

Zeno Varanini Presidente AISSA 